\title{
The Scaled Reassigned Spectrogram Adapted for Detection and Localisation of Transient Signals
}

\author{
Isabella Reinhold \\ Mathematical Statistics, \\ Centre for Mathematical Sciences, \\ Lund University, Sweden, \\ isabella@maths.lth.se
}

\author{
Josefin Starkhammar \\ Department of Biomedical Engineering, \\ Lund University, Sweden, \\ josefin.starkhammar@bme.lth.se
}

\author{
Maria Sandsten \\ Mathematical Statistics, \\ Centre for Mathematical Sciences, \\ Lund University, Sweden \\ sandsten@maths.lth.se
}

\begin{abstract}
The reassigned spectrogram can be used to improve the readability of a time-frequency representation of a nonstationary and multi-component signal. However for transient signals the reassignment needs to be adapted in order to achieve good localisation of the signal components. One approach is to scale the reassignment. This paper shows that by adapting the shape of the time window used with the spectrogram and by scaling the reassignment, perfect localisation can be achieved for a transient signal component. It is also shown that without matching the shape of the window, perfect localisation is not achieved. This is used to both identify the time-frequency centres of components in a multi-component signal, and to detect the shapes of the signal components. The scaled reassigned spectrogram with the matching shape window is shown to be able to resolve close components and works well for multi-components signals with noise. An echolocation signal from a beluga whale (Delphinapterus leucas) provides an example of how the method performs on a measured signal.
\end{abstract}

\section{INTRODUCTION}

For non-stationary signals the reassigned spectrogram (ReSpect) can improve the readability of the time-frequency representation [1], [2]. The concentration of a component is increased by reassigning mass to the centre of gravity, squeezing the signal terms to be more localised, while crossterms are reduced by a smoothing of the specific distribution. Recently, the theoretical expressions for the reassigned Gabor spectrograms of Hermite functions have been derived [3], [4]. Although the Re-Spect gives perfect localisation of linear chirps, this is not achieved for transient signals which are common in for example marine biosonar research. Transient signals can effectively be modelled by a linear combination of Hermite basis functions [5], [6], [7], [8]. Perfect localisation of a Gaussian function (first Hermite function) can be achieved by the adaptable reassignment methods, the Levenberg-Marquardt reassignment [9] and the scaled reassigned spectrogram (ScReSpect) [10].

This paper builds on the ScRe-Spect to show that perfect localisation in time and frequency can be achieved with reassignment for higher order Hermite functions. Perfect localisation is possible if the shape of the time window used with the spectrogram is matched with the shape of the signal

This work was supported by the Swedish strategic research programme eSSENCE. component. It also shows that when the matching shape window is not used, perfect localisation is not possible with the ScRe-Spect. This can be used to detect the shape of and localise the time-frequency centres of individual transient components in a non-stationary signal.

To illustrate the use of the ScRe-Spect with matching shape window this paper includes an example of an echolocation signal from a beluga whale (Delphinapterus leucas). In this field, there is a need for signal processing methods that allows for analysis of the time dependence of each frequency component within each echolocation signal [11], [12], [13].

In this paper, section II calculates the reassignment coordinates for first and second Hermite signal components with first and second Hermite time windows. The results are also extended to multi-component signals. The performance of the ScRe-Spect is evaluated in section III, by simulating transient multi-component signals with noise. Section IV shows the performance of the method on a measured echolocation signal from a dolphin. Section V concludes the paper.

\section{THE SCALED REASSIGNED SPECTROGRAM}

The spectrogram of the signal $x(t)$ using the window $h(t)$ is found from the short-time Fourier transform (STFT)

$$
S_{x}^{h}(t, \omega)=\left|F_{x}^{h}(t, \omega)\right|^{2}=\left|\int x(s) h^{*}(s-t) e^{-i \omega s} d s\right|^{2} .
$$

The Re-Spect, with reassignment to $\hat{t}_{x}$ and $\hat{\omega}_{x}$, is defined as

$$
R S_{x}^{h}(t, \omega)=\iint S_{x}^{h}(s, \xi) \delta\left(t-\hat{t}_{x}(s, \xi), \omega-\hat{\omega}_{x}(s, \xi)\right) d s d \xi
$$

where $\delta(t, \omega)$ is the two-dimensional Dirac impulse defined as $\iint f(t, \omega) \delta\left(t-t_{0}, \omega-\omega_{0}\right) d t d \omega=f\left(t_{0}, \omega_{0}\right)$. As shown in [10], the scaling factors $c_{t}$ and $c_{\omega}$ can be introduced and the reassignment coordinates can be computed as

$$
\begin{aligned}
& \hat{t}_{x}(t, \omega)=t+c_{t} \mathfrak{\Re}\left(\frac{F_{x}^{t h}(t, \omega)}{F_{x}^{h}(t, \omega)}\right), \\
& \hat{\omega}_{x}(t, \omega)=\omega-c_{\omega} \mathfrak{I}\left(\frac{F_{x}^{d h / d t}(t, \omega)}{F_{x}^{h}(t, \omega)}\right),
\end{aligned}
$$

where $\mathfrak{R}$ and $\mathfrak{I}$ are the real and imaginary parts respectively and $F_{x}^{h}, F_{x}^{t h}$ and $F_{x}^{d h / d t}$ are STFTs with different time 
windows. If $c_{t}=c_{\omega}=1$ the Re-Spect is obtained [4], [14]. Since the reassignment coordinates are calculated from STFTs using the same signal values needed for the spectrogram and only differs in the choice of time window, the computational complexity of the ScRe-Spect is not drastically increased compared the spectrogram.

\section{A. The scaled reassigned spectrogram of multi-component transient signals}

A multi-component transient signal can be modelled as a sum of Hermite functions

$$
x(t)=\sum_{k=1}^{n} a_{k} x_{k}\left(t-t_{k}\right) e^{-i \omega_{k} t},
$$

where $x_{k}(t)$ are Hermite basis functions, $t_{k}$ and $\omega_{k}$ are the time and frequency centres and $a_{k}$ the amplitudes. Due to the linearity of the Fourier transform, the reassignment vector is also linear [14]. For the calculations it is assumed that the reassignment can be calculated for each component individually. This is true for a signal with somewhat separated components, in time or frequency. The spectrogram also obeys time-frequency shift-invariance, meaning that further analysis can be restricted to signals of the form $x_{k}(t)=g_{k}(t)$ instead of $x_{k}(t)=g_{k}\left(t-t_{k}\right) e^{-i \omega_{k} t}$.

This paper analyses the unit energy Gaussian function $x_{1}(t)=\sigma^{-1 / 2} \pi^{-1 / 4} e^{-\frac{t^{2}}{2 \sigma^{2}}}$, and the unit energy second Hermite function $x_{2}(t)=2^{1 / 2} \sigma^{-3 / 2} \pi^{-1 / 4} t e^{-\frac{t^{2}}{2 \sigma^{2}}}$, as the amplitude of the signal has no effect on the reassignment coordinates, compare (3). The ScRe-Spect with perfect localisation of $x_{1}(t)$ using a Gaussian window is shown in [10]. The next section will show that the ScRe-Spect of a signal $x(t)=x_{1}(t)+x_{2}(t)$ with a Gaussian window will only give perfect localisation to $x_{1}(t)$ while the energy of $x_{2}(t)$ remains scattered, and when a second Hermite window is used, perfect localisation will be obtained for $x_{2}(t)$ but not for $x_{1}(t)$.

\section{B. Scaled reassignment with a Gaussian window}

The reassignment coordinates for $x_{1}(t)$ using a window $h_{1}(t)=x_{1}(t)$, where the window and signal have the same time length, are

$$
\begin{aligned}
\hat{t}_{x_{1}}^{h_{1}}(t, \omega) & =t-c_{t} \frac{t}{2}, \\
\hat{\omega}_{x_{1}}^{h_{1}}(t, \omega) & =\omega-c_{\omega} \frac{\omega}{2},
\end{aligned}
$$

and perfect localisation is achieved when $c_{t}=c_{\omega}=2$ [10]. To find the reassignment coordinates for $x_{2}(t)$ with $h_{1}(t)$, we need to calculate the necessary STFTs, also assuming that the time length of the signal and window are the same

$$
\begin{aligned}
& F_{x_{2}}^{h_{1}}(t, \omega)=\frac{\sqrt{2}}{\sigma^{2} \sqrt{\pi}} \int s e^{-\left(s^{2}+(s-t)^{2}\right) /\left(2 \sigma^{2}\right)} e^{-i \omega s} d s \\
& =\frac{\sqrt{2}}{\sigma^{2} \sqrt{\pi}} e^{-t^{2} /\left(2 \sigma^{2}\right)} \int s e^{-s^{2} / \sigma^{2}+\left(t / \sigma^{2}-i \omega\right) s} d s \\
& =\frac{t-i \sigma^{2} \omega}{\sqrt{2} \sigma} e^{-\left(t^{2} / \sigma^{2}+\sigma^{2} \omega^{2}+i 2 t \omega\right) / 4},
\end{aligned}
$$

$$
\begin{aligned}
& F_{x_{2}}^{t h_{1}}(t, \omega)=\frac{\sqrt{2}}{\sigma^{2} \sqrt{\pi}} \int s(s-t) e^{-\left(s^{2}+(s-t)^{2}\right) /\left(2 \sigma^{2}\right)} e^{-i \omega s} d s \\
& =\frac{2 \sigma^{2}-t^{2}-\sigma^{4} \omega^{2}}{2 \sqrt{2} \sigma} e^{-\left(t^{2} / \sigma^{2}+\sigma^{2} \omega^{2}+i 2 t \omega\right) / 4} .
\end{aligned}
$$

The derivative of $h_{1}(t)$ is $d h_{1}(t) / d t=-t h_{1}(t) / \sigma^{2}$, thus

$$
F_{x_{2}}^{d h_{1} / d t}(t, \omega)=-\frac{1}{\sigma^{2}} F_{x_{2}}^{t h_{1}}(t, \omega) .
$$

This gives the reassignment coordinates

$$
\begin{gathered}
\hat{t}_{x_{2}}^{h_{1}}(t, \omega)=t-c_{t}\left(\frac{t}{2}-\frac{\sigma^{2} t}{t^{2}+\sigma^{4} \omega^{2}}\right), \\
\hat{\omega}_{x_{2}}^{h_{1}}(t, \omega)=\omega-c_{\omega}\left(\frac{\omega}{2}-\frac{\sigma^{2} \omega}{t^{2}+\sigma^{4} \omega}\right) .
\end{gathered}
$$

It can be seen that there exist no $c_{t}$ or $c_{\omega}$ so that $\left(\hat{t}_{x_{2}}^{h_{1}}(t, \omega), \hat{\omega}_{x_{2}}^{h_{1}}(t, \omega)\right)=(0,0)$, i.e. the centre of the component, $\forall t, \omega$. Instead of perfect localisation of the component $x_{2}(t)$, the reassigned energy will be located on ellipses, which is also found for the reassigned Gabor spectrogram in [4].

\section{Scaled reassignment with a second Hermite window}

Using the second Hermite window, $h_{2}(t)=x_{2}(t)$, with the spectrogram, makes it possible to get perfect localisation to $x_{2}(t)$ with scaled reassignment. For the calculations of the reassignment coordinates it is assumed that the time length of the window is the same as for the signal,

$$
\begin{aligned}
& F_{x_{2}}^{h_{2}}(t, \omega)=\frac{2}{\sigma^{3} \sqrt{\pi}} \int s(s-t) e^{-\left(s^{2}+(s-t)^{2}\right) /\left(2 \sigma^{2}\right)} e^{-i \omega s} d s \\
& =\frac{2 \sigma^{2}-t^{2}-\sigma^{4} \omega^{2}}{2 \sigma^{2}} e^{-\left(t^{2} / \sigma^{2}+\sigma^{2} \omega^{2}+i 2 t \omega\right) / 4} \\
& F_{x_{2}}^{t h_{2}}(t, \omega)=\frac{2}{\sigma^{3} \sqrt{\pi}} \int s(s-t)^{2} e^{-\left(s^{2}+(s-t)^{2}\right) /\left(2 \sigma^{2}\right)} e^{-i \omega s} d s \\
& =\frac{w(t, \omega)-2 \sigma^{2} t-i 6 \sigma^{4} \omega}{4 \sigma^{2}} e^{-\left(t^{2} / \sigma^{2}+\sigma^{2} \omega^{2}+i 2 t \omega\right) / 4},
\end{aligned}
$$

where $w(t, \omega)=t^{3}+i \sigma^{2} t^{2} \omega+\sigma^{4} t \omega^{2}+i \sigma^{6} \omega^{3}$. Since $d h_{2} / d t=$ $h_{2}(t) / t-t h_{2}(t) / \sigma^{2}$, we get

$$
\begin{aligned}
& F_{x_{2}}^{d h_{2} / d t}(t, \omega)=\frac{2}{\sigma^{3} \sqrt{\pi}} \int s e^{-\left(s^{2}+(s-t)^{2}\right) /\left(2 \sigma^{2}\right)} e^{-i \omega s} d s \\
& -\frac{1}{\sigma^{2}} F_{x_{2}}^{t h_{2}}(t, \omega) \\
& =\frac{6 \sigma^{2} t+i 2 \sigma^{4} \omega-w(t, \omega)}{4 \sigma^{4}} e^{-\left(t^{2} / \sigma^{2}+\sigma^{2} \omega^{2}+i 2 t \omega\right) / 4} .
\end{aligned}
$$

This gives the following reassignment coordinates

$$
\begin{aligned}
\hat{t}_{x_{2}}^{h_{2}}(t, \omega) & =t-c_{t} \frac{t}{2}, \\
\hat{\omega}_{x_{2}}^{h_{2}}(t, \omega) & =\omega-c_{\omega} \frac{\omega}{2} .
\end{aligned}
$$

It can be seen that by choosing $c_{t}=c_{\omega}=2$ indeed $\left(\hat{t}_{x_{2}}^{h_{1}}(t, \omega), \hat{\omega}_{x_{2}}^{h_{1}}(t, \omega)\right)=(0,0), \forall t, \omega$ and perfect localisation in time and frequency is achieved. 
(a)

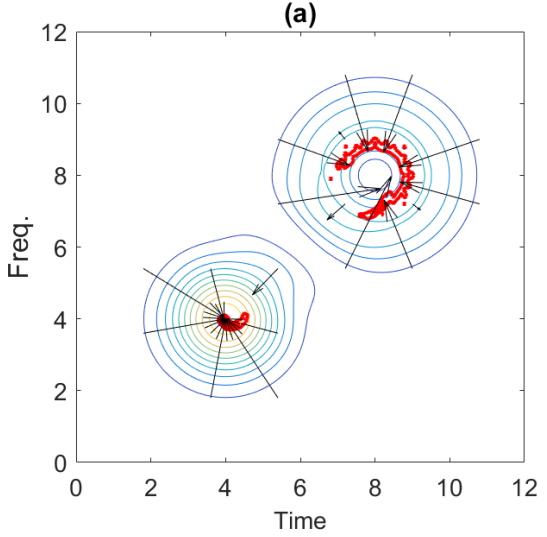

(b)

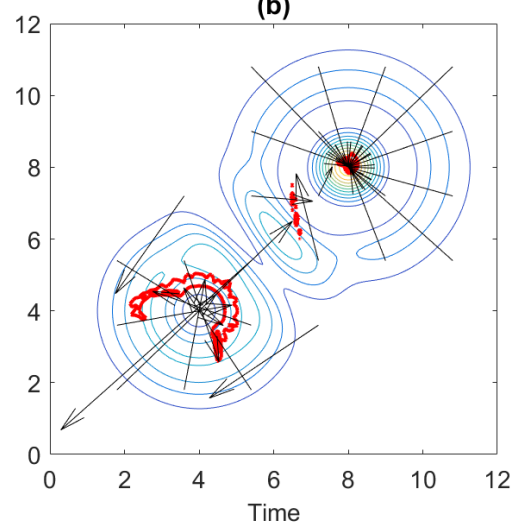

Fig. 1. Illustration of the spectrogram and the scaled reassignment of a signal with one Gaussian, $(4,4)$, and one second Hermite, $(8,8)$, signal component. The spectrogram is shown by the gradient lines. The arrows represent the reassignment of the signal energy and the red areas show the most energy dense areas in the ScRe-Spect; (a) Scaled reassignment with a Gaussian window; (b) Scaled reassignment with a second Hermite window.

Perfect time and frequency localisation is not possible when using the second Hermite window with the Gaussian component $x_{1}(t)$. This is shown by calculating the reassignment coordinates,

$$
\begin{gathered}
F_{x_{1}}^{h_{2}}(t, \omega)=-\frac{t+i \sigma^{2} \omega}{\sqrt{2} \sigma} e^{-\left(t^{2} / \sigma^{2}+\sigma^{2} \omega^{2}+i 2 t \omega\right) / 4} \\
F_{x_{1}}^{t h_{2}}(t, \omega)=\frac{2 \sigma^{2}+\left(t+i \sigma^{2} \omega\right)^{2}}{2 \sqrt{2} \sigma} e^{-\left(t^{2} / \sigma^{2}+\sigma^{2} \omega^{2}+i 2 t \omega\right) / 4} .
\end{gathered}
$$

We remind us that $d h_{2} / d t=h_{2}(t) / t-t h_{2}(t) / \sigma^{2}$, which gives

$$
F_{x_{1}}^{d h_{2} / d t}(t, \omega)=\frac{2 \sigma^{2}-\left(t+i \sigma^{2} \omega\right)^{2}}{2 \sqrt{2} \sigma^{3}} e^{-\left(t^{2} / \sigma^{2}+\sigma^{2} \omega^{2}+i 2 t \omega\right) / 4} .
$$

This gives the reassignment coordinates

$$
\begin{aligned}
& \hat{t}_{x_{1}}^{h_{2}}(t, \omega)=t-c_{t}\left(\frac{t}{2}+\frac{\sigma^{2} t}{t^{2}+\sigma^{4} \omega^{2}}\right), \\
& \hat{\omega}_{x_{1}}^{h_{2}}(t, \omega)=\omega-c_{\omega}\left(\frac{\omega}{2}+\frac{\sigma^{2} \omega}{t^{2}+\sigma^{4} \omega^{2}}\right) .
\end{aligned}
$$

It can be seen that perfect localisation is not possible, even if small values of both $\hat{t}_{x_{1}}^{h_{2}}(t, \omega)$ and $\hat{\omega}_{x_{1}}^{h_{2}}(t, \omega)$ are possible if $c_{t}=c_{\omega}=1$ and $t$ and $\omega$ are assumed not to be large, the signal energy will be located on ellipses.

\section{Detection and localisation of a multi-component signal}

The reassignment coordinates and the resulting ScRe-Spect of a multi-component signal $x(t)=a_{1} x_{1}\left(t-t_{1}\right) e^{-i 2 \cdot f_{1} \pi t}+$ $a_{2} x_{2}\left(t-t_{2}\right) e^{-i 2 \cdot f_{2} \pi t}$, where $x_{1}(t)$ is a Gaussian function and $x_{2}(t)$ a second Hermite function, can easily be calculated by a linear operation of the reassignment coordinates [14].

An illustration of the reassignment for such a multicomponent signal, with $\left(t_{1}, f_{1}\right)=(4,4),\left(t_{2}, f_{2}\right)=(8,8)$ and $c_{t}=c_{\omega}=2$, is shown in Fig. 1. The gradient lines show the spectrogram, in Fig. 1(a) with a Gaussian window and in Fig. 1(b) with a second Hermite window. The arrows show how the signal energy is reassigned and the red areas mark the most energy dense parts in the ScRe-Spect. Fig. 1(a) shows that the energy from the Gaussian component is reassigned to a small area in the centre of the component, while the energy from the second Hermite component is reassigned to a circle around the centre of the component. In Fig. 1(b) the energy from the Gaussian component is reassigned to a circle and the energy from the second Hermite component is reassigned to a small area in the centre of the component. There is also some interaction between the components resulting in some small interference after reassignment.

In Fig. 1(a) it can also be seen that some energy is moved away from the centre of the second Hermite component. This is due to that the reassignment coordinates (9) grow large when $t, \omega \rightarrow 0$ and $c_{t}=c_{\omega}=2$. This can also be seen in Fig. 1(b) for the Gaussian component. Similarly the reassignment coordinates (17) grow when $t, \omega \rightarrow 0$ and $c_{t}=c_{\omega}=2$.

\section{Simulations}

A multi-component signal with Gaussian and second Hermite components can be resolved with the ScRe-Spect using Gaussian and second Hermite time windows. This is illustrated by the simulated signal

$$
\begin{aligned}
x(n) & =x_{1}(n-80) e^{-i 2 \pi 0.14 n}+x_{2}(n-100) e^{-i 2 \pi 0.18 n} \\
& +x_{3}(n-60) e^{-i 2 \pi 0.19 n}+e(n),
\end{aligned}
$$

where $x_{1}(n)$ and $x_{2}(n)$ are Gaussian functions and $x_{3}(n)$ a second Hermite function, all with lengths around 60 samples, and $e(n)$ is white Gaussian noise, SNR $=15 \mathrm{~dB}$, where SNR is the average total signal energy to the variance of the noise. Fig. 2 shows the spectrogram and ScRe-Spect. The spectrogram and the ScRe-Spect, the illustrations are made in 3D to clearly show the difference in amplitude of the peaks, i.e. the energy density. In Fig. 2(a), showing the spectrogram with a Gaussian window, only one clear peak is visible and it is located at $(n, f)=(97,0.167)$, however in Fig. 2(c), the ScRe-Spect with a Gaussian window, two peaks are clearly 
(a)

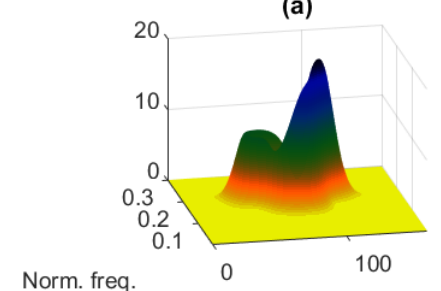

(c)

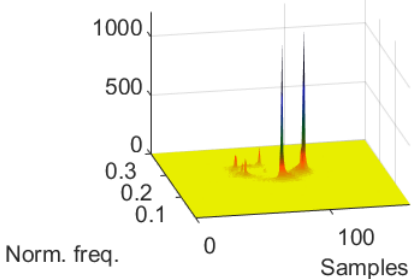

(b)

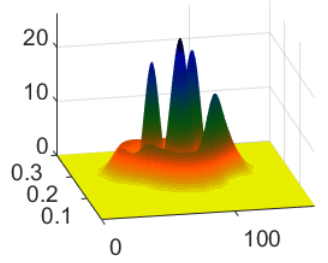

(d)

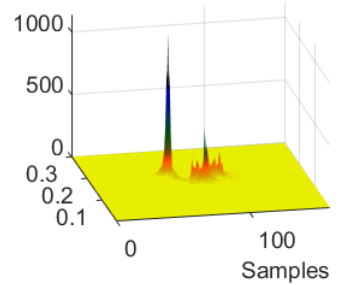

Fig. 2. Detection and time-frequency localisation of individual components in a multi-component transient signal; (a) The spectrogram with Gaussian window; (b) The spectrogram with second Hermite window; (c) The ScReSpect with Gaussian window; (d) The ScRe-Spect with second Hermite window.

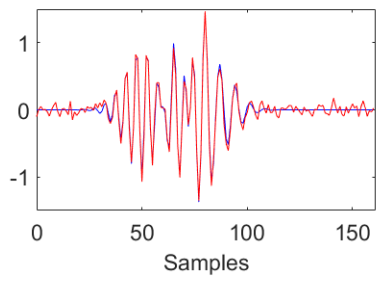

Fig. 3. Two component signal (19), $f_{1}=0.14$, with and without noise, SNR $15 \mathrm{~dB}$.

seen at $(80,0.140)$ and $(101,0.180)$, which are very close to the true centres of the two Gaussian components. Fig. 2(b), the spectrogram with a second Hermite window, shows many peaks and is hard to interpret, however Fig. 2(d) shows only one large peak at $(60,0.190)$, also present in the corresponding spectrogram, which is the true centre of the second Hermite component.

\section{A. Close components}

Detection and localisation of components becomes harder if they are close in time and frequency. For a simulated signal with two components

$$
x(n)=x_{1}(n-80) e^{-i 2 \pi f_{1} n}+x_{2}(n-60) e^{-i 2 \pi 0.2 n}+e(n),
$$

where $x_{1}(n)$ is a Gaussian function, $x_{2}(n)$ a second Hermite function and $e(n)$ is white Gaussian noise, SNR $15 \mathrm{~dB}$, we can vary the normalised frequency $f_{1}$ to change the frequency distance between the components. The components overlap in time, see Fig. 3 where $f_{1}=0.14$. We let $0.1 \leq f_{1} \leq 0.19$ and simulate 200 signals with different noise for each $f_{1}$, to find the average sample-frequency (time-frequency) centres from the ScRe-Spect for both signal components. The samplefrequency centres are obtained by finding the largest peak in (a)

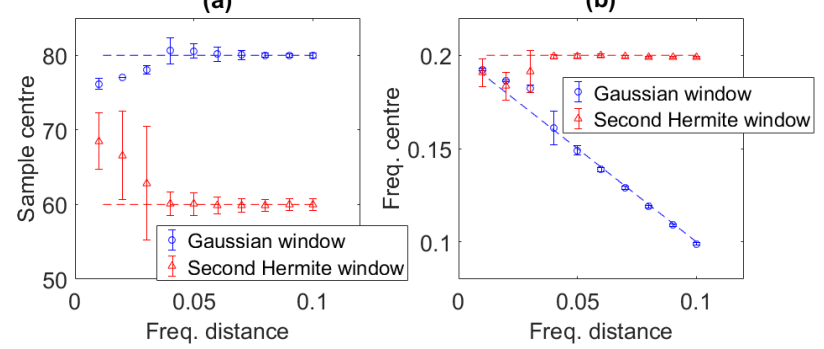

Fig. 4. The frequency distance between the two components in signal (19) is varied to get the average sample-frequency centres of the largest peaks in the ScRe-Spect with Gaussian and second Hermite window respectively, as well as a $95 \%$ confidence interval, obtained from 200 noise simulations. The true sample-frequency centres of both signal components are also marked; (a) The sample centres of the largest peaks; (b) The frequency centres of the largest peaks. (a)

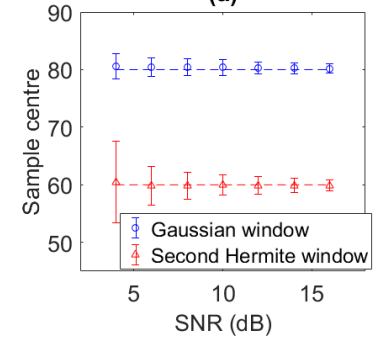

(b)

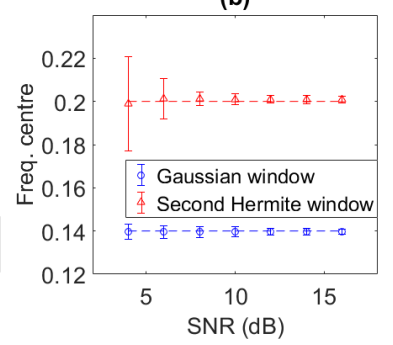

Fig. 5. The SNR of the signal (19), $f_{1}=0.14$, is varied to get the average sample-frequency centres of the largest peaks in the ScRe-Spect with Gaussian and second Hermite window respectively, as well as a 95\% confidence interval, obtained from 200 noise simulations. The true sample-frequency centres of both signal components are also marked; (a) The sample centre of the largest peaks; (b) The frequency centres of the largest peaks.

the ScRe-Spect, with a Gaussian window for the Gaussian component and a second Hermite window for the second Hermite component. The average sample centres and the $95 \%$ confidence intervals are shown in Fig. 4(a). Fig. 4(b) shows the average frequency centres and the $95 \%$ confidence intervals. It can be seen that the largest peak in the ScRe-Spect with a matched shape window accurately represents the centre of the signal component until the normalised frequency distance is only 0.03 .

\section{B. Noise sensitivity}

Detection and localisation of components also becomes harder if the signal has low SNR. For a sampled signal (19) with $f_{1}=0.14$, the variance of the white Gaussian noise $e(n)$ can be varied to evaluate the noise sensitivity of the ScRe-Spect. The SNR is varied between 2 and $16 \mathrm{~dB}$ and the number of simulations for each SNR is 200. The average sample centres and 95\% confidence intervals from the ScReSpect with matching shape window for the two components are shown in Fig. 5(a). Fig. 5(b) shows the average frequency centres and the 95\% confidence intervals. It can be seen that the method becomes unreliable for the second Hermite component around SNR $6 \mathrm{~dB}$, while the localisation of the Gaussian component remains good even for low SNR. 
(a)

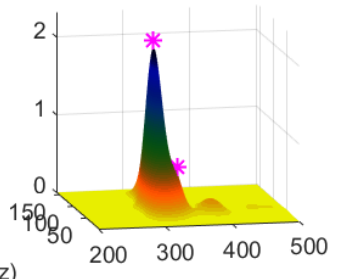

(c)

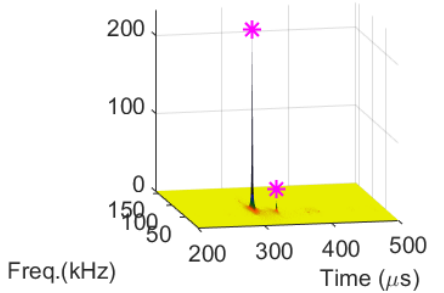

(b)

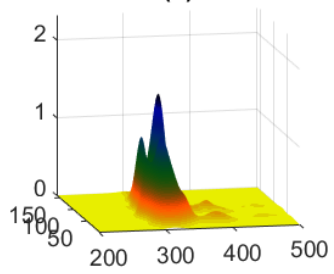

(d)

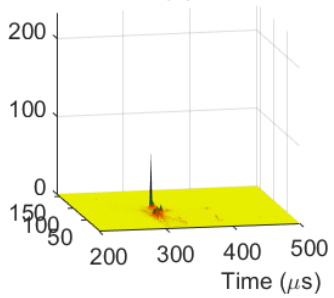

Fig. 6. Detection and time-frequency localisation of individual components in a dolphin echolocation signal; (a) The spectrogram with Gaussian window; (b) The spectrogram with second Hermite window; (c) The ScRe-Spect with Gaussian window; (d) The ScRe-Spect with second Hermite window.

\section{TRANSIENT ECHOLOCATION SIGNAL EXAMPLE}

This section provides an example of a transient echolocation signal from a beluga whale (Delphinapterus leucas). The signal is sampled with $1 \mathrm{MHz}$ and recorded by one of 47 simultaneously sampling hydrophones as described in [15]. The signal was chosen because it is recorded at the centre of the echolocation beam, based on the peak amplitude level the signal is sample by the hydrophone closest to the centre beam axis of the animal. Fig. 6(a) shows the spectrogram with a Gaussian window, Fig. 6(c) the ScRe-Spect with a Gaussian window, Fig. 6(b) the spectrogram with a second Hermite window and Fig. 6(d) the ScRe-Spect with a second Hermite window. Fig. 6(c) shows two peaks, one clear at (307 $\mu \mathrm{s}, 65 \mathrm{kHz})$ and one with smaller amplitude at $(338 \mu \mathrm{s}, 53$ $\mathrm{kHz}$ ), these peaks corresponds well to the spectrogram in Fig. 6(a). In Fig. 6(d) the signal energy is more scattered even if peaks appear, the peaks also do not correspond well to the spectrogram in Fig. 6(b). This suggests that the signal components in the dolphin echolocation signal more closely resembles Gaussian functions than second Hermite functions.

With additional measurements, this method could determine if the larger and smaller Gaussian like components originate from two locations within the sound generation mechanism of the dolphin or are the result of internal reflection of the sound wave. Assuming an approximate sound velocity of $1490 \mathrm{~m} / \mathrm{s}$ in the tissue, the time separation of the components corresponds to a total difference in sound wave travel distance of approximately $4.6 \mathrm{~cm}$.

\section{CONCLUSIONS}

It is shown that perfect time-frequency localisation of a Gaussian and second Hermite transient signal component can be achieved by the ScRe-Spect using a matching shape time window. If a time window which do not match the shape of the signal component is used, the component energy is instead scattered in ellipses around the time-frequency centre of the component. It is shown that this can be used to find the timefrequency centres and the shapes of the individual transient signal components within a multi-component signal.

The results from simulated multi-component signals with noise show that the ScRe-Spect can resolve and correctly identify the time-frequency centres and component shapes even if the signal components are close in time and frequency. The ScRe-Spect is also robust to noise disturbances. The performance is evaluated on a measured dolphin echolocation signal, which gives good time-frequency localisation of what seems to be two Gaussian-like signal components.

\section{REFERENCES}

[1] K. Kodera, C. de Villedary, and R. Gendrin, "A new method for the numerical analysis of nonstationary signals," Physics of the Earth \& Planetary Interiors, vol. 12, pp. 142-150, 1976.

[2] F. Auger and P. Flandrin, "Improving the readability of time-frequency and time-scale representations by the reassignment method," IEEE Trans. on Signal Processing, vol. 43, pp. 1068-1089, May 1995.

[3] F. Auger, E. Chassande-Mottin, and P. Flandrin, "On phase-magnitude relationships in the short-time Fourier transform," IEEE Signal Processing Letters, vol. 19, no. 5, pp. 267-270, May 2012.

[4] P. Flandrin, "A note on reassigned Gabor spectrograms of Hermite functions," J Fourier Analysis and Applications, vol. 19, no. 2, pp. 285-295, 2013, doi 10.1007/s00041-012-9253-2.

[5] A.I. Rasiah, R. Togneri, and Y. Attikiouzel, "Modeling 1-D signals using Hermite basis functions," in IEEE Proc.-Vis. Image Signal Process. IEEE, 1997, vol. 144, pp. 345-354.

[6] T. H. Linh, S. Osowski, and M. Stodolski, "On-line heart beat recognition using Hermite polynomials and neuro-fuzzy network," IEEE Trans. on Instrumentation and Measurement, vol. 52, no. 4, pp. 12241231, August 2003.

[7] B.N. Li, M.C. Dong, and M.I. Vai, "Modeling cardiovascular physiological signals using adaptive Hermite and wavelet basis functions," IET Signal Processing, vol. 4, no. 5, pp. 588-597, 2010.

[8] R. Ma, Z. Huang L. Shi, and Y. Zhou, "EMP signal reconstruction using associated-Hermite orthogonal functions," IEEE Trans. on Electromagnetic Compatibility, vol. 64, no. 6, pp. 1383-1390, March 2016.

[9] F. Auger, E. Chassande-Mottin, and P. Flandrin, "Making reassignment adjustable: The Levenberg-Marquardt approach," in 2012 IEEE International Conference on Acoustics, Speech and Signal Processing (ICASSP), March 2012, pp. 3889-3892.

[10] M. Hansson-Sandsten and J. Brynolfsson, "The scaled reassigned spectrogram with perfect localization for estimation of Gaussian functions," IEEE Signal Processing Letters, vol. 22, no. 1, pp. 100-104, January 2015.

[11] C. Capus, Y. Pailhas, K. Brown, D.M. Lane, P. Moore, and D. Houser, "Bio-inspired wideband sonar signals based on observations of the bottlenose dolphin (Tursiops truncatus)," J. Acoust. Soc. Am., vol. 121, no. 1, pp. 594-604, 2007.

[12] J. Starkhammar and M. Hansson-Sandsten, "Evaluation of seven timefrequency representation algorithms applied to broadband echolocation signals," Advances in Acoustics and Vibration, vol. 2015, pp. 1-13, 2015.

[13] J. Starkhammar, I. Reinhold, P. Moore, D. Houser, and M. Sandsten, "Intra-click time-frequency patterns across the echolocation beam of a beluga whale," The Journal of the Acoustical Society of America, vol. 140, no. 4, pp. 3239-3239, 2016.

[14] E. Chassande-Mottin, F. Auger, and P. Flandrin, "Reassignment," in Time-Frequency Analysis, F. Hlawatsch and F. Auger, Eds., chapter 9, pp. 249-277. U.K.: ISTE, London, 2008.

[15] J. Starkhammar, M. Amundin, J. Nilsson, T. Jansson, S. Kuczaj, M. Almqvist, and H.W. Persson, "47-channel burst-mode recording hydrophone system enabling measurements of the dynamic echolocation behavior of free-swimming dolphins," J Acoust Soc Am., vol. 126, no. 3, pp. 959-962, 2009. 